SSC11-III-7

\title{
STP-SIV: Lessons Learned Through the First Two Standard Interface Vehicles
}

\author{
Mr. Michael Pierce, Dr. David Kaufman \\ Ball Aerospace \& Technologies Corp. \\ 1600 Commerce St, Boulder, CO 80301 (303) 533-4101, (303) 533-5262 \\ mpierce@ball.com, dakaufma@ball.com
}

\author{
Mr. Kenneth Reese \\ DOD Space Test Program, Space and Missile Systems Center, Space Development and Test Directorate \\ 3548 Aberdeen Avenue, Kirtland AFB, NM 87117 (505) 846-7730 \\ Kenneth.Reese@kirtland.af.mil
}

\begin{abstract}
Spacecraft standards can enable dramatic reduction in the cost, schedule, and risk of spaceflight. After the first build of a standard spacecraft the non-recurring engineering is complete which allows rapid, cost-effective production of subsequent units. Spacecraft standards can increase the supplier base by encouraging multiple organizations to build components, subsystems, or spacecraft according to the same standard. Interface standards promote rapid and lowrisk integration. The Space Development \& Test Directorate (SDTD) of the USAF Space and Missile Systems Center (SMC) developed standards for the Space Test Program-Standard Interface Vehicle (STP-SIV) program with prime contractor Ball Aerospace \& Technologies Corp. STP-SIV is a small spacecraft bus with well-defined and documented standard interfaces to the payload, ground, and launch vehicle. STP-SIV is designed to provide affordable, repeatable, and reliable space access to the science and technology (S\&T) community. STP-SIV provides the space community well-defined standard spacecraft (SC) to-payload (PL) interface on which to base PL designs for rapid mission formation. Rather than designing unique SC for each payload; the standards provide adaptable interfaces to accommodate a range of payloads.

With the first STP-SIV spacecraft, STPSat-2, operating in-orbit since November 2010 and the second vehicle, STPat-3, bus integration completed in January 2010, two data sets are available to quantitatively examine:

- $\quad$ Bus integration efficiency with commercially available components

- $\quad$ Payload integration and test timelines achieved with standardization

- $\quad$ Improvements in efficiency from vehicle \#1 to \#2

- $\quad$ Relative cost savings from vehicle \#1 to \#2

- $\quad$ Lessons learned, successes, and drawbacks of the standardization approach

These topics will be explored using examples and data from both vehicle projects, offering the reader insight into the challenges and successes surrounding the topic. The paper will also describe how the lessons learned have contributed to program efficiencies for the second vehicle, recommendations for future improvements, and how the STP- SIV approach could be further evolved to meet the aggressive demands of rapid, low-cost, responsive space.
\end{abstract}



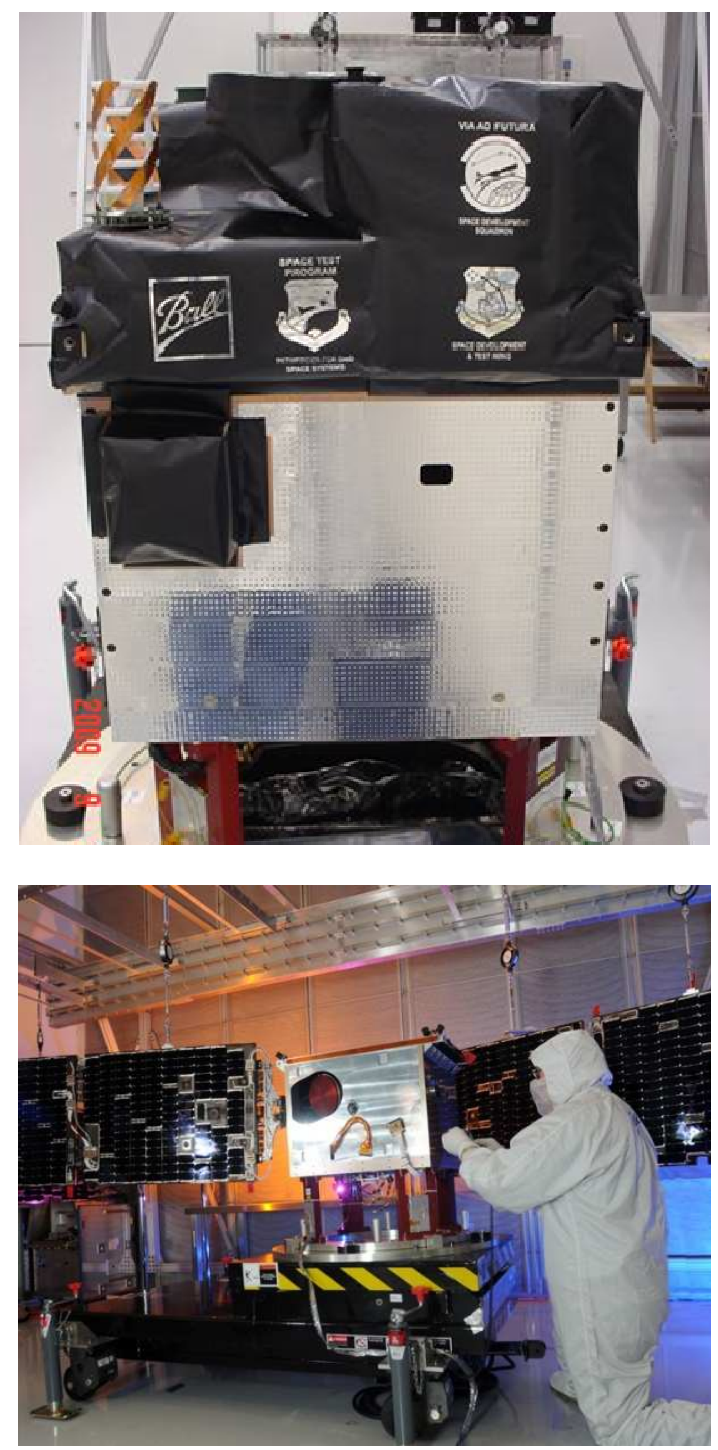

Figure 1 - STPSat-2 (top) and STPSat-3 (bottom) are the first two spacecraft in the STP-SIV product line, designed to increase access to space for small payloads via standardization and lower costs

\section{INTRODUCTION}

The STP-SIV program is the latest of several attempts across the industry to develop a standard space vehicle capable of satisfying a range of mission requirements and thereby provide a low-cost, low risk, flexible space platform suitable for multiple applications with minimal non-recurring engineering. The goal of this acquisition was to develop a spacecraft product line capable of meeting the Department of Defense Space Test Program's need for affordable and rapid access to low earth orbit through the next decade. Responsiveness was not an explicit program goal, but the standardization approach leads to inherent responsiveness or the architecture as described in this paper. STPSat-2 (Figure 1) was the first spacecraft in the STP-SIV product line, launched on 19 Nov 2010 and now operating successfully on-orbit.

Table 1 shows a top level summary of the STP-SIV capabilities. The standard design and payload interface provide mission flexibility, enable operation over a wide range of orbits, and allow for launch on a variety of Launch Vehicles (LVs). STP-SIV is designed for small payloads (70 kg payload mass) and is compatible with the cost-effective Evolved Expendable Launch Vehicle (EELV) Secondary Payload Adapter (ESPA) and the Minotaur-IV Multi-Payload Adaptor (MPA).

\begin{tabular}{|c|c|}
\hline \multicolumn{2}{|c|}{ Spacecraft Capabilities } \\
\hline Orbit Altitude & 400 to $850 \mathrm{~km}$ \\
\hline Orbit Inclination & 0 deg to $98.8 \mathrm{deg}$ \\
\hline Launch Mass (Payload + Bus) & $\leq 180 \mathrm{~kg}$ \\
\hline LV Compatibility & $\begin{array}{l}\text { Delta IV ESPA, Atlas V ESPA, } \\
\text { Minotaur I, Minotaur IV, } \\
\text { Pegasus }\end{array}$ \\
\hline SV Lifetime & 5 years \\
\hline Stabilization Method & 3 -axis \\
\hline Pointing Modes & $\begin{array}{l}\text { Nadir, surface point tracking, } \\
\text { inertial point, payload sun point, } \\
\text { safe }\end{array}$ \\
\hline Attitude Knowledge & $0.02 \operatorname{deg} 3 \sigma($ goal $0.02 \operatorname{deg} 3 \sigma)$ \\
\hline Attitude Control & $0.1 \operatorname{deg} 3 \sigma($ goal $0.03 \operatorname{deg} 3 \sigma)$ \\
\hline Bus Voltage & $28 \mathrm{~V} \pm 6 \mathrm{~V}$ \\
\hline Communication Frequency & AFSCN Compatible \\
\hline Command Rate & 2 kbps uplink \\
\hline Telemetry Rate & 2 Mbps downlink \\
\hline On-Board Data Storage & $16 \mathrm{Gbit}$ \\
\hline \multicolumn{2}{|c|}{ Payload Accommodation } \\
\hline Payload mass & $70 \mathrm{~kg}$ (Total) \\
\hline $\begin{array}{l}\text { Payload Orbit Average Power } \\
\text { (OAP) }\end{array}$ & $>100$ Watts (Total) \\
\hline Number of Payloads & Up to four \\
\hline Payload Data Handling & $\begin{array}{l}\text { Up to } 2.0 \mathrm{Mbps} \text { from each } \\
\text { payload }\end{array}$ \\
\hline $\begin{array}{l}\text { Payload Command/Data } \\
\text { Interface }\end{array}$ & RS-422, discrete $\mathrm{I} / \mathrm{O}$, analog \\
\hline
\end{tabular}

Table 1 - STP-SIV is designed to accommodate a wide range of payload and mission requirements and is capable of launching on multiple LVs 
The standard interfaces were conceived with the intent of increasing spaceflight opportunities, lowering costs, and reducing risk for missions and potential customers. The program sponsor, Space Development and Test Directorate (SDTD), shares a fundamental goal with other government organizations to lower costs and increase the speed of access to space. The STP-SIV is an important step in achieving this goal.

As part of the STP-SIV contract, Ball Aerospace defined a robust standard payload interface that addresses all aspects of spacecraft and payload interaction. These include thermal, mechanical, electrical, power, and data interface specifications along with minimum requirements for payload testing. The standard interface provides broad resources to accommodate a wide variety of payload needs. The details of the interface, design and testing requirements along with guidance to the payload providers are documented in the STP-SIV Payload User's Guide (PLUG) [1]. For individual payloads, Ball and the payload developer must agree to the specifics of how each payload will use the interfaces. This nonambiguous agreement is documented in an Interface Control Document (ICD) and is the single source for interface requirements. The ICD specifies not only the requirement but also the method of requirement verification and provides traceability to verification artifacts.

\section{INTERFACE STANDARDIZATION AS AN ENABLER FOR RESPONSIVE ACCESS TO SPACE}

By its charter, SDTD "develops, tests, and evaluates Air Force space systems, executes advanced space development and demonstration projects, and rapidly transitions capabilities to the warfighter."

A key element of the SDTD/SDSG mission is to develop advanced space systems that demonstrate DoD S\&T and new capabilities for our warfighters. The costeffective approach enabled by the STP-SIV allows SDTD to increase the number of spaceflight opportunities for the S\&T community and thus helps accomplish the SDTD/SDS mission. With cost effectiveness and increased access to space as key objectives, the STP-SIV design is a natural fit for deployment as a responsive space asset as a consequence of its standardization and flexibility.

STP-SIV has four external interfaces (Figure 2): payload, launch vehicle, Air Force Satellite Control Network (AFSCN) and the mission ops complex. Interfaces with the launch vehicle are kept simple by design and are intended to be as common as possible for all the potential launch vehicles. The AFSCN and mission operations facility interfaces were developed for STPSat-2, and minimal payload-specific changes are expected for STPSat-3 and future STP-SIV missions. The payload interface is defined and documented in the PLUG, but the specific implementation changes for every mission within the configurable standard.

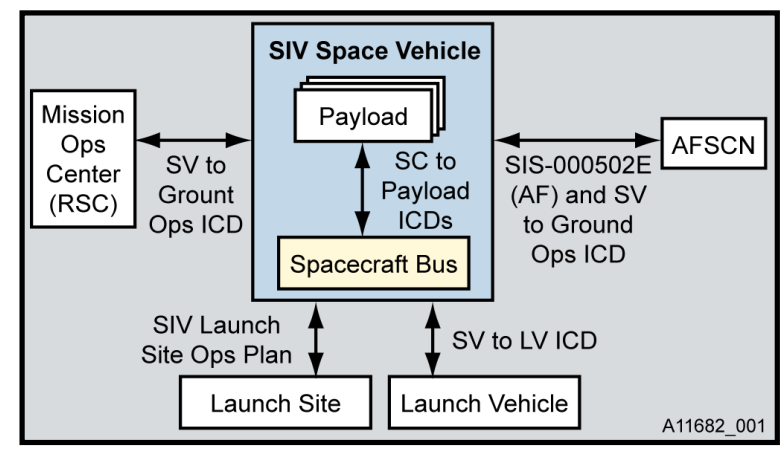

\section{Figure 2 - External interfaces with the STP-SIV spacecraft are rigorously defined.}

\section{Space Vehicle to Launch Vehicle Interface}

STP-SIV is designed to launch on a variety of LVs, including Pegasus, Minotaur I, Minotaur IV, and the ESPA on either Atlas V or Delta IV (compatibility with Falcon 9 is expected). This flexibility maximizes launch manifest opportunities as a secondary payload or as a rideshare partner and is a key element in satisfying SDTD's objective of maximizing its spaceflight opportunities. The spacecraft was designed to be bounded by the environments for the above launch vehicles to ensure generic compatibility. STP-SIV was designed to meet the ESPA volume (Figure 3) as it was the most constraining. Furthermore, STP-SIV is designed to be powered off prior to integration with the launch vehicle and requires only safety monitoring of the battery and the ability to trickle charge for periods as long as 90 days. This keeps the number of interfaces required from the launch vehicle to a minimum and aids in compatibility with multiple platforms. By designing in compatibility with a range of candidate launch vehicles and qualifying the design for the maximum enveloping environment, the STP-SIV enables responsive launch to urgent needs. A STP-SIV with a high priority payload could take advantage of a launch of opportunity on any launch vehicle or could be manifested quickly on a launch vehicle prepared in advance for a responsive launch.

\section{Spacecraft to AFSCN Interface}

The interface between STP-SIV and ground facilities is controlled by two documents; an Interface Control Document (ICD) and the Standardized Interface Specification for the Air Force Satellite Control 
Network (AFSCN), SIS-000502C. SIS-000502 defines the types of service provided by the AFSCN and the design requirements for the SV radio frequency (RF) system. The ICD describes the specific characteristics employed by the space vehicle RF system to show compliance with these requirements. This includes defining the exact operating frequency, subcarrier frequencies, modulation scheme, etc. Telecom frequencies have been selected and pre-approved by the relevant licensing organization for use on future SIVs. This eliminates risk of the sometime time-consuming approval process and enables transponder production to proceed without interruption. With predetermined frequencies, a transponder or full SIV could be produced and "on the shelf" for deployment in a responsive space application since there is no uncertainty in the mission telecom frequency and no risk of changes.

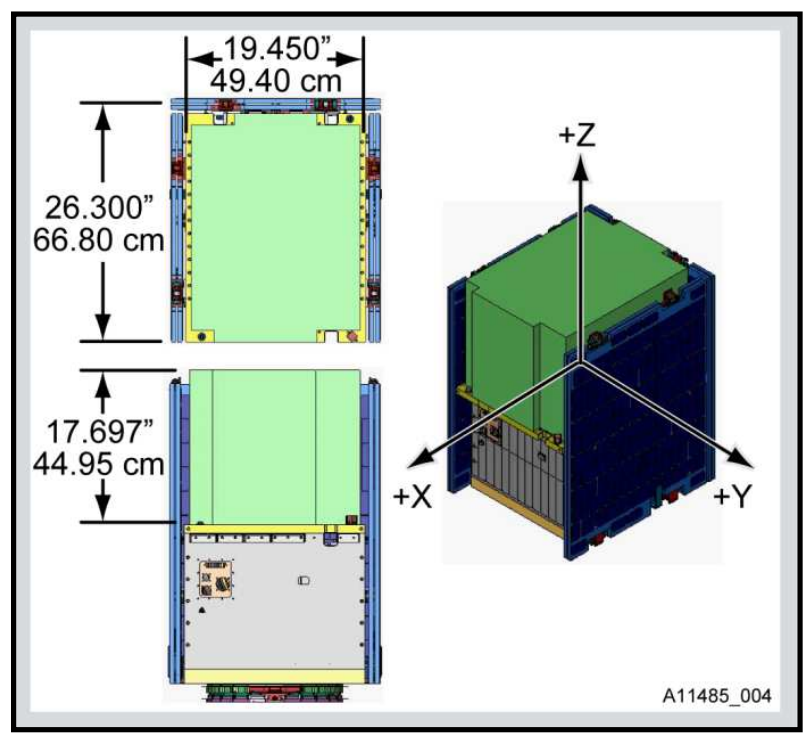

Figure 3 - Payload envelope, shown in green, provides clear definition for payload packaging to ensure compatibility with variety of LVs

\section{Spacecraft to Mission Operations Facility Interface}

STPSat-2 mission operations are performed at the Research, Development, Test \& Evaluation (RDT\&E) Support Complex (RSC) at Kirtland AFB, NM. The interface between the STP-SIV and mission operations complex is governed by the SV to ground ops ICD. By flying STPSat-3 and future SIVs on the same ground system, recurring development is limited to mission unique requirements from the payload. By reusing the spacecraft telemetry formatting, the downlink of payload data is unchanged and only processing of the mission data is new. Space vehicle commanding is the same with payload unique commands encapsulated within standard command formatting. In a responsive situation, mission tailoring for each possible payload suite could be developed in advance of the mission and implemented as part of payload integration when the mission need is identified.

\section{Spacecraft to Payload Interface}

In classic space programs, the payload is developed in advance of or in parallel with the spacecraft bus, and the SV-PL interface is unique to one program. STPSIV defines and thoroughly documents a standard payload interface capable of supporting mechanical, electrical, thermal, and data transfer needs common to many payloads. By providing a well defined interface standard, payload providers and responsive space mission designers have sufficient information to proceed in parallel with designs of many payload types for a range of possible missions (Figure 4). Payloads designed to the standard become effectively interchangeable such that a single STP-SIV bus can be responsive to many missions. No longer must the payload be manifested prior to spacecraft design. In fact, for STPSat-3, the spacecraft components were ordered in March 2009 and a payload manifest decision was not finalized until May 2010.

To further streamline spacecraft-payload integration, the STP-SIV program developed a spacecraft simulator that is representative of the flight interface. High fidelity simulators can be expensive and in many cases are not developed because of the cost and schedule impacts to the program. By using a standardized interface, the STP-SIV program is able to make a onetime investment in the simulator that has been reused numerous times on STPSat-2 and STPSat-3 and will be further employed as future SIV missions are developed. The simulator consists of an Engineering Model of the payload interface electronics and runs actual flight software. The user interface runs the same Streamlink ${ }^{\mathrm{TM}}$ software used in space vehicle integration so payload command and control scripts can be exercised prior to payload delivery to Ball for spacecraft integration. By testing the payloads with the simulator prior to delivery, interface issues can be quickly and efficiently addressed at the payload developer's facility where they have more resources.

The responsive potential of the early interface verification approach was demonstrated during the STPSat-2 payload integration phase when the three STPSat-2 payloads were successfully integrated in less than 4 days in part due to the effective use of the simulator in verifying electrical and data interfaces and developing payload test scripts used during integration. The same approach was employed for STPSat-3 and results of the second flight integration of a STP-SIV 
payload suite are expected to be available by the time this paper is presented at RS2011.

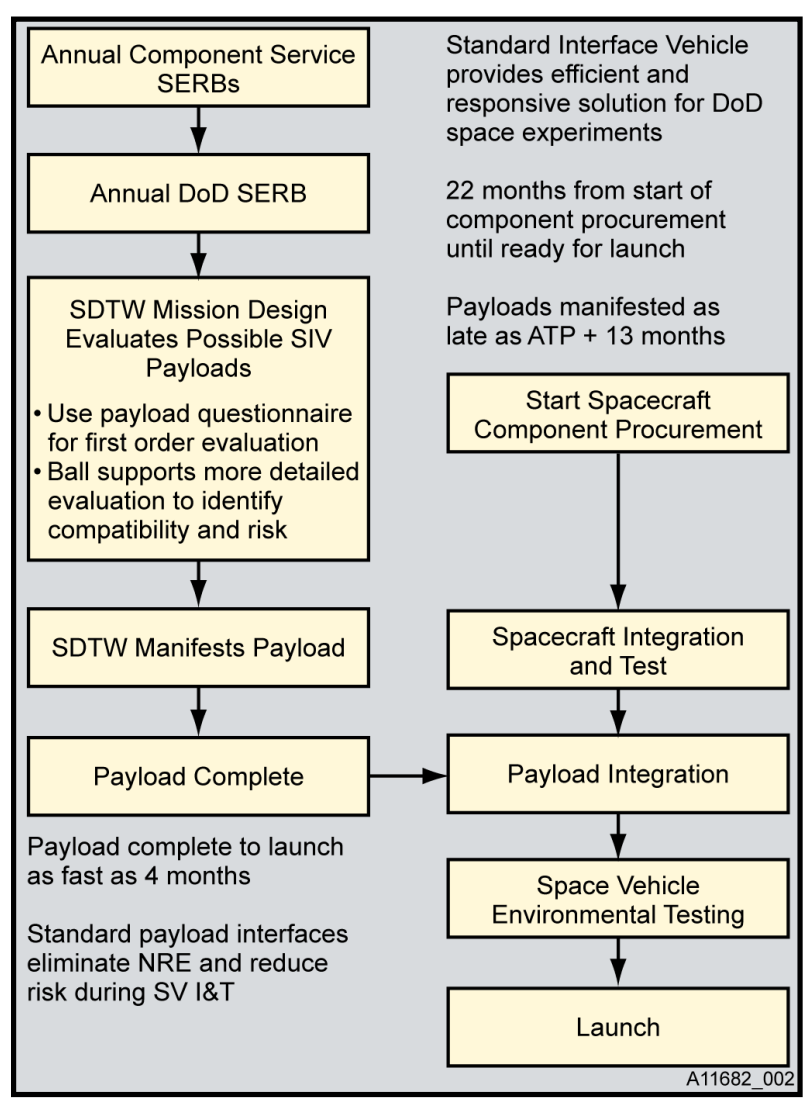

Figure 4 - Standard interfaces allow development of multiple payloads for a single spacecraft.

With the demonstrated rapid integration success, the STP-SIV spacecraft may now be considered as a standard commodity, much like other standard elements of the infrastructure supporting military space. With a compressed space vehicle development timeline, users have more flexibility to respond to the changing needs of the military and leverage emerging payloads designed to the interface standard and launch opportunities as they become available.

\section{RESPONSIVE CONTRACTING - THE STP- SIV IDIQ MODEL}

Much of the emphasis on responsive space architectures is focused on rapid integration, launch, and deployment. However, the contracting process can be a source of delays in traditional one-of-a-kind space projects. A well designed contract structure can enable significant advantages for achieving responsive space.

The STP-SIV program is structured as an Indefinite Delivery Indefinite Quantity (IDIQ) contract between the Government and the Contractor with several Contract Line Items Numbers (CLINs) available for special studies; long lead component procurement; space vehicle production, system testing, launch and commissioning support; and post-commissioning mission operations support. This arrangement enables rapid response to Government needs without protracted contract negotiations. When a need arises, the Government and contractor jointly develop a Statement of Work (SOW) for the activities to be performed, and a proposal is prepared by the Contractor for Government evaluation, negotiation, and authorization.

In the case of special studies, the SOW and proposal are typically brief. The special studies CLIN includes prenegotiated labor rates that streamline the proposal evaluation and authorization process. With this arrangement, authorization can be expedited in as little as 4 weeks from initial identification of the need for a study including SOW agreement, proposal preparation, and Government evaluation and authorization. Most studies have been undertaken to assess accommodation of candidate payload suites on the STP-SIV bus, and the results of the study may result in additional studies or in the initiation of the next space vehicle. Performing studies allows SDTD to make informed decisions in advance of authorizing further work.

A new STP-SIV space vehicle program would typically be initiated through purchase of long lead components. The STP-SIV long lead components are contracted via a firm fixed price (FFP) CLIN that provides the Government cost certainty and allows the contractor to implement the program with a streamlined management approach because of the reduced financial reporting requirements. Because of the standard SIV bus design, little new NRE is needed with each recurring spacecraft build, so long lead component selections, specifications, Mission Assurance documents, ICDs, and subcontractor SOWs are in place to enable rapid execution. As with the special studies, the SOW and proposal for purchasing long lead components can be relatively simple since all decisions about parts quality, performance, delivery schedules, and acceptance process have been agreed to by the Government, Contractor, and Subcontractors in advance. An order for long lead components can be authorized in less than 60 days from the Government RFP to ATP. The actual performance for STPSat-3 long lead components was less than 90 days from the SDTD's decision to start the vehicle. When compared to a standard new space program in which the selection process can take more than a year, the STP-SIV IDIQ and FFP CLIN for long lead components provides an advantage for space responsiveness.

Advance contracting for preset mission elements can also enable responsiveness. The STP-SIV system 
design supports advance contracting for the entire bus element. Because the STP-SIV vehicle is designed to a set of enveloping mission and interface requirements, it can support any suite of up to four payloads that meets the interface standard, it is able to meet performance specifications in any LEO orbit $(400 \mathrm{~km}-850 \mathrm{~km})$ without design changes and may launch on any of several launch vehicles without re-qualification of the design. This design robustness allows tremendous flexibility in managing bus acquisitions, payload suite groupings, orbit selection, and launch manifest decisions. The bus element can be produced in parallel with or in advance of mission selection or a previously produced bus element and could be repurposed for a new mission. This capability was employed on STPSat3 when the bus was started before the payload suite, orbit, and launch vehicle were selected. When the suite of payloads was selected a year later, no bus design changes were required.

As a consequence of the minimal NRE associated with the STP-SIV standard spacecraft design, the program staff for follow-on vehicles can be minimized during production of the long lead components. As an example, the STPSat-3 System Engineering and Integration \& Test activities (another separate CLIN) were intentionally postponed until midway through component production and after payload selection was complete. This contracting approach saved significant cost for the government as it reduced the total staffing required over the life of the program.

Government collaboration is another promising path to contracting responsiveness. As part of STP, SDTD actively solicits partnerships with Government, commercial and international organizations seeking access to space. The SDTD Reimbursable Payload program enables partner organizations to access SDTD's expertise in space vehicle development, launch vehicle integration, mission design, and ground systems as a reimbursable service. Partner organizations may arrange with SDTD for independent free-flyer missions, launch rideshares, or hosted payloads on SDTD spacecraft. Through this service, an organization seeking responsive access to space has ready access to the STP-SIV design and the existing IDIQ contract. This cross-collaborative approach could be employed throughout the responsive space community to provide all potential users with access to enabling responsive space capabilities regardless of which organization originally sponsored the development.

\section{RESPONSIVE INTEGRATION \& TEST, LAUNCH AND MISSION OPERATIONS WITH A STANDARD SPACECRAFT}

Integration and test starts with software qualification. For a new platform, significant effort is invested in developing new software or porting existing software to a new platform. Proper documentation and qualification of that software is a significant effort and most often is on the critical path leading into integration and test (I\&T) activities. Flight software development and testing is a common source of program delays. In many cases, I\&T is started without the complete software capability and software updates are introduced later in the program. This leads to further program risk and $\mathrm{I} \& \mathrm{~T}$ inefficiencies as software issues are discovered and workarounds are developed while waiting for software changes. Subsequent retesting leads to lengthened schedules. This was certainly the case on STPSat-2. With standardization, software changes on subsequent units are limited to mission unique requirements, if any, and incorporation of any lessons learned from previous spacecraft. Flight software testing on subsequent spacecraft can leverage the sizeable investment made on the first unit and a fraction of the original development cost and program risk is reduced by having a mature software baseline available at the start of I\&T.

As with the design effort, standardization leads to significant cost savings and responsive schedules with efficiencies realized in bus and vehicle level integration and test. Significant non-recurring engineering is invested in development of integration procedures and system test planning for the first unit. Many of these procedures are used only once in the I\&T flow. Specifications and procurement of GSE adds even more cost and schedule to typical programs with savings realized only on subsequent builds if change can be minimized.

In spite of detailed planning, there will always be lessons learned the first time through these procedures as the engineers and test personnel learn the system as they execute the test the first time. Traditional programs include these learning curve inefficiencies in their accepted baseline, or realize schedule challenges if the learning curve is not properly accounted for. As an example from STPSat-2, the first time the limited performance test procedure was run, it took two days to complete the testing. This procedure was run several times during system test to characterize performance during key steps such as before and after moves associated with individual environment test, between vibe axes and during SV thermal vacuum testing. As lessons learned were incorporated and the I\&T team became more familiar with the test, the duration was 
reduced from 2 days to 2 hours. I\&T consists of many procedures that are run a single time. For recurring builds, reuse of those procedures can realize the same types of gains on subsequent vehicles, significantly reducing the overall test schedule. Incorporation of lessons learned also leads to a more robust test program that can identify non-conformances earlier in the test flow.

Previous qualification reduces the risk of design issues being uncovered late in the program. Focus is on changes from previous builds. The recurring risk gets reduced to workmanship. With appropriate screening and testing at the component levels and incorporation of lessons learned earlier in the test flow, the likelihood of significant schedule impacts is much reduced. .

The STPSat-2 schedule was 16 months from the start of bus integration until the SV was complete and ready to ship to the launch site. STPSat-3 completed bus integration in 2.5 months and is on track to complete space vehicle test activities with an equivalent elapsed time of less than 9.5 months to completion once delays outside the control of the spacecraft bus flow are accounted for.

The STP-SIV program provides responsive access to space while maintaining a conservative integration and test program. Bus integration consists of thorough interface verification, safe-to-mate checks and baseline component performance testing during component integration. Space vehicle testing follows a MIL-STD$1540 \mathrm{E}$ protoqualification test program. Even with a traditional test program and risk posture consistent with a Class B/C mission, a credible 4 month schedule from payload delivery to launch can be achieved.

With a recurring build, options to further accelerate that schedule can be entertained with an acceptance of some increased risk.

- Single axis workmanship vibration test

- $\quad$ Reduction in number of system level thermal vacuum cycles and elimination of one or more thermal balance test.

- $\quad$ Tailoring of EMI/EMC testing. STPSat-3 has already eliminated radiated susceptibility (RS) testing based on previous qualification of the spacecraft bus to the specified environment. As a baseline, payloads were already doing RS testing at their level. Limiting system level EMI-EMC testing to radiated emissions and selfcompatibility testing reduced the schedule by a week.

- Initial baseline performance testing can be significantly reduced because performance has already been characterized on previous builds. Some increased level of risk is accepted because issues may not be identified until after environments.

Mission ops preparation also is another aspect of the program that can realize significant savings when leveraging a standard design. STPSat-2 developed a mature space vehicle handbook, on-orbit handbook $(\mathrm{OOH})$ and ICDs with the ground element. By keeping a common spacecraft bus design, changes from one mission to the next are limited to payload unique operations. With standard and flexible interfaces, even the payload unique operations remain similar leaving the changes mostly up to specific tasking of the space vehicle and payload unique commanding that is largely left in the hands of the payload providers and transparent to the operators.

On STPSat-2, a significant learning curve was noted through the three mission rehearsals and one mission dress rehearsal. Both spacecraft operations and payload activities were new to the mission operators. With a standard bus design, spacecraft operations will remain the same limiting changes to payload operations. Risk of operator error is significantly reduced because the system is familiar, commands and telemetry are the same and contingency procedures are engrained from multiple builds.

Typical launch interface negotiations, range safety reviews and analysis often starts two years or more prior to the actual launch date. A standard spacecraft such as STP-SIV, with documented interfaces, minimal services required of the launch vehicle, and mature, correlated models to start with, provides opportunities to significantly reducing this schedule and specifically reduce the lead time required from a manifest decision. Like the spacecraft itself, the launch vehicle takes time to procure, integrate and test. However, with standard interfaces, much of that activity could take place prior to a specific manifest decision being made. Work that is unique to specific missions could conceivably be brought into line with the shortened space vehicle test activities providing mission definition to launch in less than 6 months if the payload, spacecraft and launch vehicle hardware meets certain levels of standardization and is ready for the next steps of integration. Recent definition of ESPA standard services is taking a step towards defining such interface standards for the launch vehicle and has the potential to provide launch flexibility much later in the launch interface cycle.

STP-SIV has a mature Missile System Pre-launch Safety Package (MSPSP) that has been reviewed and accepted for the STPSat-2 program. These documents typically take considerable time to develop and iterate 
through multiple review cycles. By maintaining a standard design, changes are limited to the payloads which for this class spacecraft are typically benign to the launch environment. With the spacecraft powered off prior to launch vehicle integration and remaining powered off until after separation, the STP-SIV design meets the criteria of secondary and rideshare payloads of 'do no harm to the primary'.

\section{ECONOMIES OF A STANDARD SPACECRAFT DESIGN}

Achieving effective space responsiveness depends on reducing costs, streamlining schedules, and increasing volume production and therefore mission utility. While significant savings can be realized by standardization with architecture like STP-SIV, certain limitations must be overcome and careful design of a responsive space program itself can increase the impact that standardization has on the cost of each space vehicle. Programmatic cost drivers that can be mitigated for recurring standard vehicles include spacecraft components acquisition, program timing for continuity, and leveraging investment in Non-Recurring Engineering (NRE).

\section{Components Procurement Strategies for Cost Control and Obsolescence Mitigation}

Bus subsystem components make up a significant fraction of the total cost of a high-quality spacecraft bus like STP-SIV. A standard bus design naturally drives reduced component costs for follow-on vehicles by using the same components for each vehicle. As described above, stable component specifications, SOWs, quality requirements, and standard test plans give the purchaser and supplier a firm and well understood design target, manufacturing flow, and set of acceptance criteria for each component. Both purchaser and supplier reap cost benefits from reuse of documentation, lessons learned, and low NRE involved in designing, assembling and testing the follow-on units. Overall program schedule can be reduced because component procurement can start rapidly and meaningful reviews can be held with focus on only the changes from previous procurement.

However, a second and often more significant savings can be realized through volume production of multiple shipsets of components. If several units are ordered simultaneously, the supplier can capitalize on efficiencies that can be passed on to the purchaser. These efficiencies include shared project management resources, parallel processing of fabricated parts, reduction in minimum buy expenses for procured piece parts, shared use of assembly and test resources, amortization of staff learning curve across multiple units and shared Quality engineering of common lots of EEE parts. As an example, STPSat-3 realized approximately $20 \%$ savings on its components by leveraging the investment of NRE from STPSat-2. Since the two programs were separated by nearly 3 years, STPSat-3 was not able to benefit from block buy savings, which could have yielded up to an additional $24 \%$ savings based on supplier quotations requested at the time of the STPSat-3 Long lead components proposal.

These multiple-unit efficiencies are only available for units ordered at the same time. Unfortunately, simultaneous orders of multiple components are not commonly feasible within restricted Government funding profiles allocated on an annual basis. The Government's vision for Responsive Space offers the opportunity to change this reality. Since component production consumes many months of schedule, Responsive Space requires some stockpiling of components in advance of the need, with the ORS Rapid Response Space Works as one example. The advanced planning necessary to build up a component base offers mission managers the opportunity to execute block purchases of components on a regular schedule for improved program cost effectiveness, responsiveness to urgent mission needs, and total value to the government. Block purchases also insulate the program against parts obsolescence affects as suppliers can plan for obsolescence by purchasing parts available at-risk parts and can amortize unavoidable obsolescence upgrades over several units.

Design for a wide range of orbits and the standardization of the bus to payload interfaces makes such an acquisition strategy feasible in a standardization model like STP-SIV since bus production can occur independent of payload availability and launch opportunity. Long term storage of bus components or a fully integrated bus is also feasible since the STP-SIV bus can be stored nearly indefinitely with certain exceptions (battery life). Periodic battery replacements can be planned to enable a bus that is ready when the mission need and opportunity arise.

\section{Recurring Program Phasing for Cost Optimization}

For a recurring spacecraft program, components procurement schedules are the main driver of the program I\&T schedule since high value space components take one year or more to produce. One year is much longer than required to prepare for integration of a recurring space vehicle, so waiting for components could significantly increase the total cost of the program by extending program office and system engineering staffing by 6-8 months longer than 
required. STP-SIV's approach to manage this affect on the second vehicle has been to initiate the long lead components production as a separate firm fixed price (FFP) program as noted above. This enables the contractor to keep a limited staff to manage the purchase and production of the standard components. As a fixed price program, the management of the program can be drastically reduced because the FFP has fewer deliverables and may be executed for maximum efficiency via the contractor's internal processes. The Government and the contractor share the savings generated through leaner program execution. In trade for forgoing the typical detailed insight into the cost performance of the program, the Government realizes minimal cost risk inherent to a FFP. While assuming the risk of a FFP program, the Contractor benefits from the prospect of higher profitability if it is able to execute the program efficiently. That risk is more acceptable for the second space vehicle and beyond because the more risky NRE has been completed and subcontractor performance is better understood.

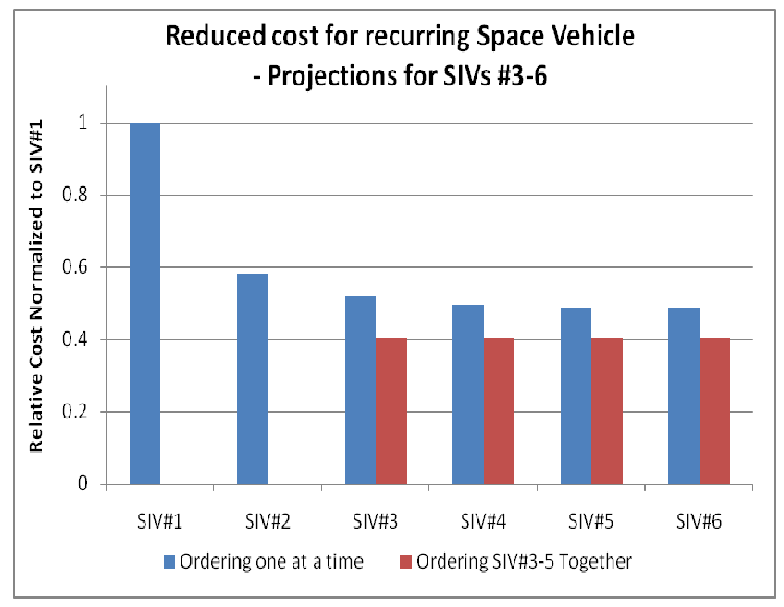

Figure 5 - STPSat-3 costs through SV completion exhibit more the $\mathbf{4 0 \%}$ savings from STPSat-2.

Another STP-SIV strategy for improving cost performance and compressing schedule was buying the longest lead piece parts even further in advance. For a modest investment of approximately $\$ 100 \mathrm{~K}$, the program purchased 5 ship sets of frequency-dependent parts for the transponder and power and data transfer slip rings for the solar array drive. Stocking these parts allowed the program to cut the delivery time of the two longest lead components by 2 months each. The resulting cost savings gained through schedule compression of STPSat-3 more than recouped the entire initial investment.

\section{NRE Investment in Standardization}

A key feature of the STP-SIV that improves its attractiveness as a responsive space asset is its design for a wide range of payloads, LEO orbits, and LVs without design changes. This approach required designing to an enveloping set of challenging requirements that drove NRE costs higher than for a spacecraft designed for any single combination of mission orbit, launch vehicle, and payload suite. The designs of several subsystems in particular were driven by the expanded requirements of a standard vehicle including Electrical Power (EPS), Attitude Determination and Control (ADCS), Flight Software (FSW), Telecommunications and Thermal Control. Additional design and analysis effort was required to design the system capable of operating in the large parameter space and component performance requirements were elevated to meet the system requirements. Most notably, the EPS design involved a greatly expanded analysis of orbits, inclinations and operational modes. Even the process of defining the driving analysis cases required additional engineering time. The results drove the need for a higher capacity battery and a larger solar array featuring two fixed wings and an articulated wing. The ADCS was required to support additional operational modes and the FSW design needed to incorporate the unique control features. The Star Tracker and Sun Sensor placement design needed to satisfy many different vehicle orientations relative to the earth and sun. Similar to the EPS analysis, the STP-SIV thermal design needed to consider a much larger trade space for a standardized vehicle than for a spacecraft designed for a single mission. The analysis of sun angles, orbits, a range of payload heat loading, and heater power requirements drove a significantly greater engineering effort. Telecommunications analysis, too, was driven by the array of vehicle orbits and orientations. The investment in standardization is being recovered in the second STP-SIV, STPSat-3. With STPSat-3 approximately $70 \%$ complete, the $\mathrm{SV}$ is forecast to cost less than $60 \%$ of the actual cost of STPSat-2, which included all the NRE investment (Figure 5). Future vehicles have the promise of further savings as process improvements and learning curve improve program efficiency. Simultaneous order of several SIVs would further improve affordability as the figure illustrates. A key part of the incremental cost realization from vehicle to vehicle has been staff continuity and investment in documentation updates for future vehicles. Throughout STPSat-2 and STPSat-3 programs, lessons learned were thoroughly documented by continuing staff. Rather than documenting lessons learned in a separate format, the lessons have been incorporated in working program documentation such as verification documents, subcontract specifications and SOWs, I\&T procedures, and manufacturing instructions. The evolving documentation allowed the STPSat-3 program to realize 
the predicted high level of efficiency even as the program staff changed over time.

\section{CONCLUSION}

The STP-SIV program has developed a standard vehicle that provides an option for cost-effective, capable, flexible spaceflight for the Responsive Space community. The program is now employing lessons learned from the first vehicle program, STPSat-2, launched in November 2011 to the second vehicle, STPSat-3, which was completed through bus integration in January 2011. The key features discussed include;

- Bus and payload interface standardization is an enabler for responsiveness to a variety of space missions with a single bus

- $\quad$ Establishing and enforcing standard interfaces can reap dividends in reduced NRE build-to-build, a compressed production schedule, and rapid response to changing priorities

- Careful design of the contract can enable significant schedule compression during program initiation and can be used to realize cost efficiencies

- Significant cost savings and schedule reduction can be realized through design standardization that leads to reuse of integration and test procedures, familiarity of test operations and incorporation of lessons learned leading to lower risk and greater efficiency.

- $\quad$ Launch and mission ops can be streamlined on recurring builds with familiarity of activities and reuse of documentation.

- Responsiveness of a standard bus can be improved through purchasing multiple components simultaneously and ordering targeted long lead elements in advance to reduce component procurement costs and schedules.

\section{REFERENCES}

[1] Space Test Program-Standard Interface Vehicle (STP-SIV) Payload Users Guide, Revision C, June 2008 